\title{
ESTUDO FITOQUÍMICO, BOTÂNICO E DAS PROPRIEDADES ANTIMICROBIANAS DE Pterocaulon interuptum DC. (ASTERACEAE)
}

\section{PHYTOCHEMICAL, BOTANICAL STUDIES AND ANTI MICROBIAL PROPERTIES FROM Pterocaulon intemuptum DC. (ASTERACEAE)}

\author{
HEEMANN, A. C. W.1; MIGUEL, O. G. ${ }^{2}$
}

\author{
${ }^{1}$ Mestre em Ciências Famacêuticas pelo Programa de pós graduação em Ciências Farmacêuticas - UFPR -Dissertação \\ ${ }^{2}$ Orientador. Prof. Dr. Obdulio Gomes Miguel - obdulio@ufpr.br
}

\section{RESUMO}

No presente trabalho foram realizados estudos sobre a espécie Pterocaulon intemuptum DC., Asteraceae, cujas partes aéreas foram submetidas a estudo fitoquímico, sendo o processo extrativo por Soxhlet, o qual apresenta melhor rendimento e rapidez, isolando-se e caracterizando-se cinco compostos: sabandinol (cumarina), quercetina (flavonol), taxifolina 7-O-prenilada (di-hidroflavonol), estigmasterol (esteróide) e 3-O-acetil taraxasterol (esteróide). Do óleo essencial foram caracterizados oito compostos: a-tujeno; 1,4,6-trimetil-5,6-dihidronaftaleno; 3-heptanona; acetaldeído benzênico; alil cilohexano; carvacrol (metil éter); orto-cimeno e safrol. Estudos botânicos foram realizados para determinação das caracteństicas anatômicas e possível comparação com estudos anteriores. A fração do extrato alcoólico avaliada não apresentou atividade inibitória frente a cepas de Escherichia coli, Pseudomonas aenuginosa e Staphylococcus aureus.

Palavras-chave: Pterocaulon intemuptum DC.; Asteraceae; fitoquímica; botânica; óleo essencial; propriedades antimic robianas.

\section{ABSTRACT}

In the present paper was realized studies about Pterocaulon intemuptum DC., Asteraceae family, which aerial parts were submitted to botanic al study to obtain anatomic characteristic sand possibility to compare with others studies. It was conducted the phytochemical studies and the extractive process was developed by Soxhlet, which presented a betterperformance and speed. It was isolated five compounds: sabandinol (coumarin), quercetin (flavonol), 7-0-prenyl taxifolin (dihydroxyflavonol), stigmasterol (steroid) and 3-O-acetyl taraxasterol (steroid). From essential oil was identified eight compounds: a-thujene; 1,4,6-trimethyl-5,6-dihydronaphtalene; 3-heptanone; benzenic acetaldeid; alil ciclohexane; carvacrol (methyl ether); ortho-cimene and safrol. The fraction available from alcoholic extract wasn't active like anti microbial in the microorganisms species Escherichia coli, Pseudomonas aenuginosa e Staphylococcus aureus.

Key words: Pterocaulon intemuptum DC.; Asteraceae family; phytochemistry; botanical; essential oil; anti mic robial properties. 